\title{
Development of 3D Beam-Beam Simulation for the Tevatron*
}

\author{
E. Stern, J. Amundson, P. Spentzouris, A. Valishev, Fermilab, Batavia, IL 60510, USA \\ J. Qiang, R. Ryne, LBNL, Berkeley, CA 94720, USA
}

\begin{abstract}
We present status of development of a 3D Beam-Beam simulation code for simulating the Fermilab Tevatron collider. The essential features of the code are 3D particlein-cell Poisson solver for calculating the Beam-Beam electromagnetic interactions with additional modules for linear optics, machine impedance and chromaticity, and multiple bunch tracking. The simulations match synchrobetatron oscillations measured at the VEPP-2M collider. The impedance calculations show beam instability development consistent with analytic expressions.
\end{abstract}

\section{MOTIVATION}

The Fermilab Tevatron [1] is a $p-\bar{p}$ collider in which counter-rotating beams of 36 bunches of both particle species share a common vacuum pipe. For high-energy physics operations, the beams collide head-on at two specific interation points (IPs), but parasitic collisions occur at 136 other locations. The intentional and unintentional beam-beam interactions produce coherent motion of multiple bunches. Impedance and beam-beam effects in extended length bunches couple longitudinal motion to transverse degrees of freedom. The accelerating RF fields in the longitudinal direction provide an energy source that when coupled to the transverse motion can produce instabilities. Understanding the interplay between all these effects requires developing a comprehensive simulation. We will present progress in the development of such a simulation, with validation of the subcomponents against observed effects and analytic calculations.

\section{BEAMBEAM3D CODE}

The Poisson solver in the BeamBeam3d code is described in references [2]. Bunches of macro-particles in two beams are generated with a random distribution in phase space. The accelerator ring is conceptually divided into arcs with potential interaction points at the ends of the arcs. The optics of each arc is modeled with a $6 \times 6$ linear map that transforms the phase space $\left\{x, x^{\prime}, y, y^{\prime}, z, \delta\right\}$ coordinates of each macroparticle from one end of the arc to the other. There is a significant amount of coupling between the horizontal and vertical transverse coordinates in the Tevatron. For our Tevatron simulations, the maps were calculated using coupled lattice functions citeOptim1 obtained by fitting a model [4] of beam element configuration to beam measurements. The synchrotron motion is put in

\footnotetext{
* work supported by the SciDAC program of the U.S. Department of Energy
}

as a sinusoidal oscillation with the periodicity of the machine synchrotron tune. The Tevatron includes electrostatic separators to generate a helical trajectory for the oppositely charged beams. The mean beam offset at the IP is included on the Poisson field solver calculation.

Different particle bunches are individually tracked through the accelerator. They interact with each other with the pattern and locations that they would have in the real accelerator.

The impedance model uses the dipole component of resistive wall wakefields [5]. Each beam bunch is divided longitudinally into slices containing approximately equal numbers of particles. As each bunch is transported through an arc, particles in a slice receive a $\Delta x^{\prime}$ kick from the wake field induced by the dipole moment of the particles in forward slices. The kick from slice $i$ is:

$$
\Delta \vec{p}_{\perp} / p=\frac{2}{\pi b^{3}} \sqrt{\frac{c}{\sigma}} \frac{N_{i}<\vec{r}_{i}>}{\beta \gamma} \frac{L}{\sqrt{z_{i}}}=W_{0} L<\vec{r}>
$$

The length of the arc is $L, N_{i}$ is the number of particles in slice $i, z_{i}$ is the longitudinal distance between the particle and the slice that induces the wake, $\vec{r}_{i}$ is the mean transverse position of particles in slice $i, b$ is the pipe radius, $c$ is the speed of light, $\sigma$ is the conductivity of the beam pipe and $\beta \gamma$ are Lorentz factors of the beam.

\section{SYNCHRO-BETATRON COMPARISONS}

Coherent synchro-betatron modes, observed in an experiment at the VEPP-2M $500 \mathrm{MeV} e^{+} e^{-}$collider and described in reference [6] are an unambiguous marker of beam-beam interactions and provide sensitive tool for evaluating calculational models. In the case of a colliding beam accelerator where the longitudinal bunch length and the beta function are of comparable size, particles at different $z$ positions are coupled through the electromagnetic interaction with the opposing beam. Coherent modes develop with tune spectra that are sums and differences of the betatron and synchrotron tune. The tune shifts for different modes have a characteristic evolution with beam-beam parameter $\xi=N r_{0} / 4 \pi \epsilon$, in which $N$ is the number of particles, $r_{0}$ is the classical electromagnetic radius, and $\epsilon$ is the emittance.

We simulated the VEPP-2M collider using CourantSnyder uncoupled maps. The horizontal emittance in the VEPP-2M beam is much larger than the vertical emittance. The bunch length $(4 \mathrm{~cm})$ is comparable to $\beta_{y}^{*}=6 \mathrm{~cm}$ so we expect to see synchrobetatron modes. In order to excite synchrobetatron modes, we set an initial $y$ offset of one beam sigma approximately matching the experimental conditions. 


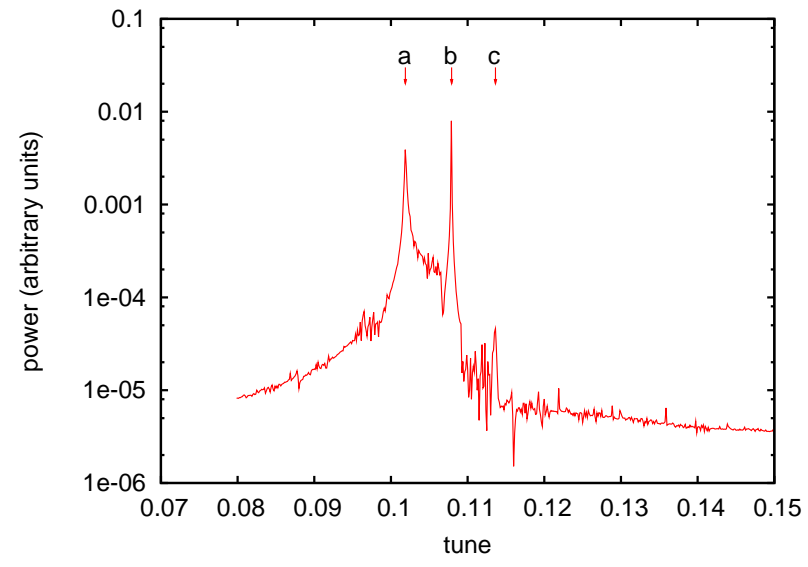

Figure 1: Simulated mode spectra in the VEPP-2M collider with $\xi=.008$ showing synchrobetatron modes. The line indicated by a) is the base tune, b) is the first synchrobetatron mode, $\mathrm{c}$ ) is the beam-beam $\pi$ mode.

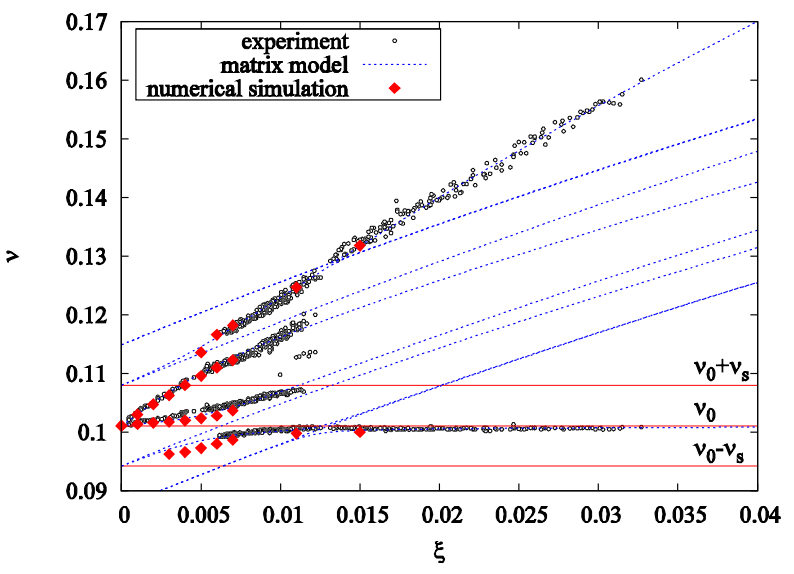

Figure 2: The diamonds show simulated synchrobetatron modes as a function of beam-beam parameter $\xi$ (diamonds) of observed modes (points).

Longitudinal effects of the beam-beam interaction were simulated by dividing the bunch into six slices. At the interaction point, bunches drift through each other. Particles in overlapping slices are subject to a transverse beam-beam force calculated by applying a kick from the electric field determined by solving the 2D Poisson equation for particles in the overlapping beam slice. Fig. 1 shows an example spectrum from a simulation with the three mode peaks indicated.

In Fig. 2, the BeamBeam3d simulation of synchrobetatron modes as a function of $\xi$ is plotted on top of experimental data from VEPP-2M. As can be seen, there is good agreement between the observation and simulation giving us confidence in the beam-beam calculation.

\section{IMPEDANCE TESTS}

Wakefields or equivalently impedance in an accelerator with a conducting vacuum pipe gives rise to well known instabilities. Our aim in this section is to demonstrate that the wakefield model in BeamBeam3d quantitatively reproduced those theoretically and experimentally well understood phenomena. The strong head-tail instability examined by Chao [5] arises in extended length bunches in the presence of wakefields. For any particular accelerator optical and geometric parameters, there is an an intensity threshold, above which the beam becomes unstable.

We divide the Tevatron ring into 12 arcs. The betatron tune of the machine is on the order of 20.5, so the apparent tune from the Fourier spectrum sampling 12 times per turn is the the actual tune modulo 12. Using a pipe radius of $3 \mathrm{~cm}$ and a bunch length of $20 \mathrm{~cm}$ produces the spectrum shown in Fig. 3a for a bunch of $4 \cdot 10^{12}$ protons at $150 \mathrm{GeV}$. The two mode peaks are clearly evident. The upper peak is betatron frequency 8.574 shifted down by the wakefield. The lower peak is the lower synchrobetatron mode $8.574-.007$ shifted upwards by the wakefield.

In Fig. 3b, we show the evolution of the two modes as a function of beam intensity. We show two sets of curves for two slice and six slice wakefield calculations. In Chao's derivation, instability develops when the quantity $\Upsilon=\pi N r_{0} W_{0} L / 4(2 \pi)^{2} \gamma \nu_{\beta} \nu_{s} \geq 2$ where $\nu_{\beta}$ and $\nu_{s}$ are the betatron and synchrotron tunes, $L$ is the length of the ring, and $W_{0}$ is the wakefield factor defined previously. With the parameters of this simulation, the formula predicts instability development at intensities of about $9 \cdot 10^{12}$ particles. However this formula assumes an airbag particle distribution instead of a Gaussian distribution, and a constant wakefield that only effects the bunch tail instead of the more realistic situation of the field decreasing with distance.

The growth rate per turn of dipole motion at the threshold of strong head-tail instability has a parabolic dependence on beam intensity. The wakefield calculation also reproduces this feature as shown in Fig. 4.

Chromaticity interacts with impedance to cause a different head-tail instability. Going arc-by-arc, chromaticity results in an additional phase advance $\delta \mu_{x(y)}=$ $\mu_{0} \xi_{x(y)} \Delta p / p$ where $\xi_{x(y)}$ is the chromaticity for $x$ (or $y$ ) and $\mu_{0}$ is the design phase advance for the arc. In transporting particles through an arc in the presence of chromaticity, a new coupled map is calculated for each particle using the coupled lattice functions at the beginning and ending of the arc with the additional phase advance.

We simulated a range of beam intensities and chromaticity values. The two particle model, and the more general Vlasov equation calculation [5] indicate that the growth rate scales by the head-tail phase $2 \pi \xi \nu_{\beta} / c \eta, \eta$ is the slip factor of the machine and $\hat{z}$ is a measure of the bunch length. This phase is a measure of how much betatron oscillations are affected by chromaticity over the length of the bunch. When the growth rate is normalized 

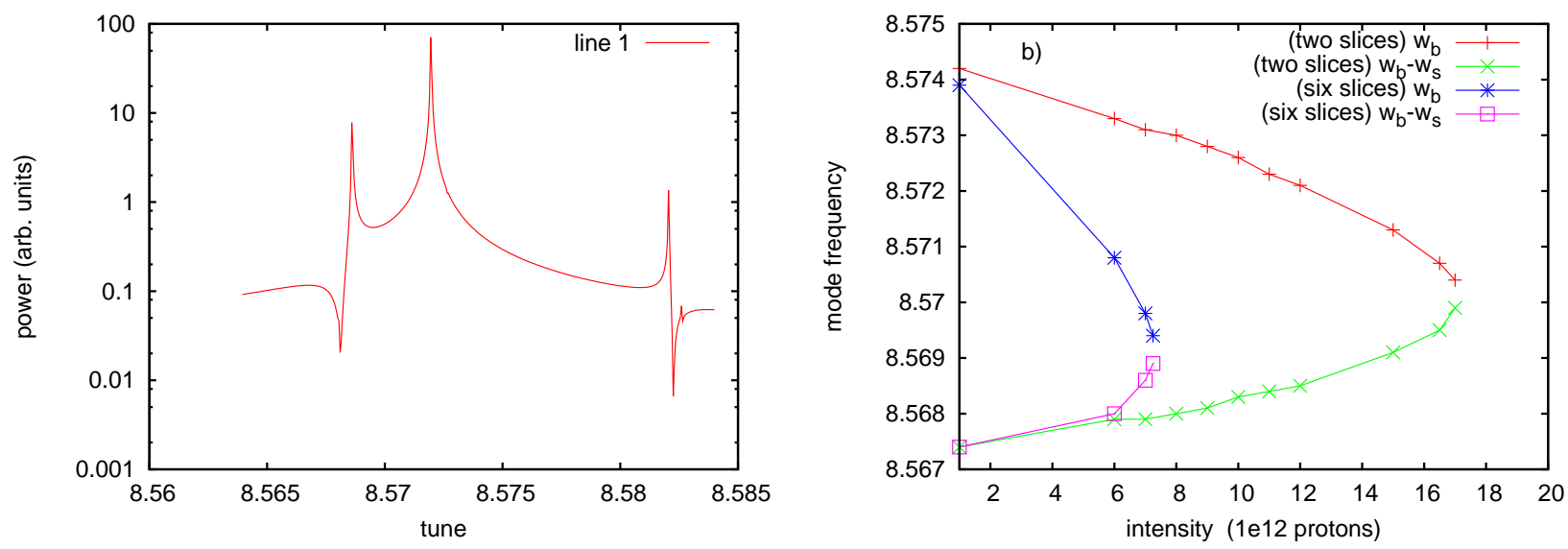

Figure 3: a) Simulated spectrum of a two slice bunch in the presence of wakefields showing two modes. b) Evolution of the mode frequencies as a function of beam intensity showing the two modes coming to a common frequency. The simulations are shown for two slice and six slice wakefield calculation.

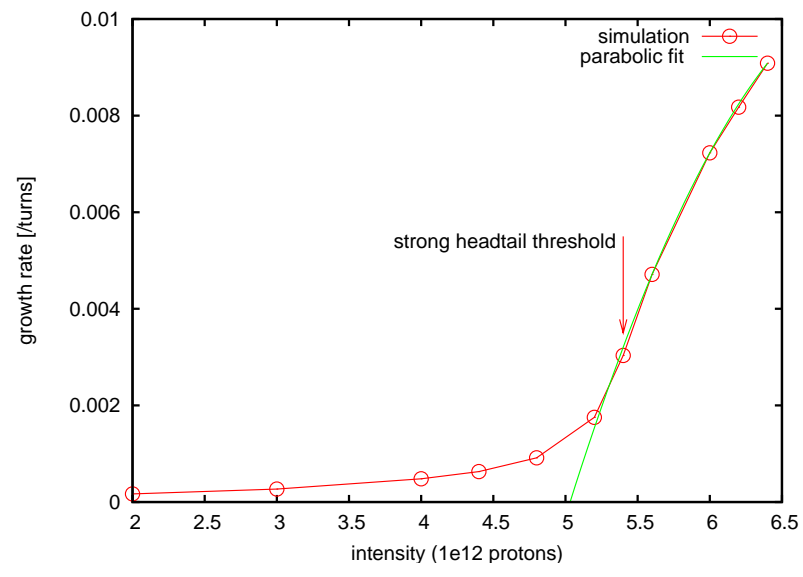

Figure 4: The growth rate of dipole motion in the simulated accelerator with impedance as a function of beam intensity as the strong head-tail threshold is reached.

by $N r_{0} W_{0} / 2 \pi \beta \gamma \nu_{\beta}$ which includes the beam intensity and geometric factors, we expect a universal dependence of normalized growth versus head-tail phase that begins linearly with head-tail phase and peaks around -1 . Fig. 5 shows the simulated growth rate at three intensities with a range of chromaticites from -.001 to -0.5 to get headtail phases in the 0 to -1 range. The normalized curves are nearly identical and peak close to head-tail phase of unity. The deviation from a universal curve is again due to differences between the idealized model and detailed simulation.

\section{SUMMARY}

We have validated the correctness of modules that simulate beam-beam interactions, resistive wall impedance, and chromaticity in a combined accelerator model. With multiple bunch tracking, we are now able to investigate their contributions to instability development.

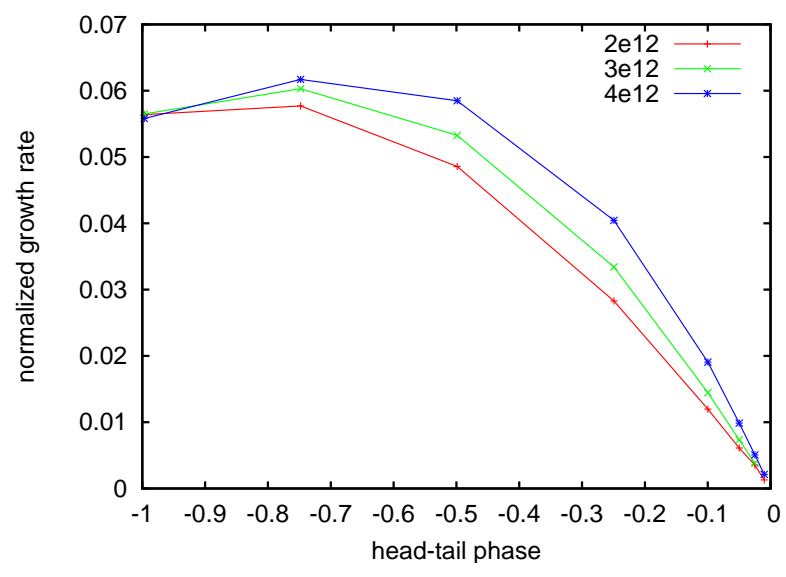

Figure 5: The normalized growth rate of dipole motion in the simulated accelerator with impedance and chromaticity as a function of head-tail phase at three beam intensities.

\section{REFERENCES}

[1] Run II handbook, http://www-bd.fnal.gov/runII

[2] J. Qiang, M.A. Furman, R.D. Ryne, J.Comp.Phys., 198 (2004), pp. 278-294, J. Qiang, M.A. Furman, R.D. Ryne, Phys. Rev. ST Accel. Beams, 104402 (2002).

[3] V. Lebedev, http://www-bdnew.fnal.gov/pbar/ organizationalchart/lebedev/OptiM/optim.htm

[4] A. Valishev et al., "Progress with Collision Optics of the Fermilab Tevatron Collider", EPAC06, Edinburgh, Scotland, 2006

[5] A. Chao, Physics of Collective Beam Instabilities in High Energy Accelerators., pp. 56-60, 178-187, 333-360 John Wiley and Sons, Inc., (1993)

[6] I.N. Nesterenko, E.A. Perevedentsev, A.A. Valishev, Phys.Rev.E, 65, 056502 (2002) 